\title{
Welchen Einfluss haben verschiedene Vatertypen auf den Verlauf der Symptombelastung ihrer Kinder? Eine Längsschnittuntersuchung an 14- bis 23-Jährigen
}

\author{
Which influence do different types of fathers exert on their offspring's \\ psychopathological development? A longitudinal study on 14 to 23 year \\ olds
}

\section{Zusammenfassung:}

In einer Längsschnittstudie wurde der Einfluss dreier Vatertypen auf die Symptombelastung ihrer Kinder im Jugend- und im jungen Erwachsenenalter analysiert. An einer Stichprobe aus 213 Probanden wurde die Symptombelastung zu fünf Messzeitpunkten im Jugendalter (Youth SelfReport) und im jungen Erwachsenenalter (Young Adult Self-Report) untersucht. Die Ergebnisse der Studie zeigen erhöhte Werte in der internalisierenden Symptombelastung der weiblichen im Vergleich zu den männlichen Probanden. Des Weiteren weisen die Ergebnisse auf erhebliche Probleme bei jungen Erwachsenen, welche ihren Vater im Jugendalter als zunehmend negativ oder distanziert beschrieben haben, hin. In diesen beiden Gruppen zeigte sich zu allen Messzeitpunkten eine höhere Symptombelastung als in der Gruppe der jungen Erwachsenen, welche ihren Vater im Jugendalter als normativ beschrieben haben.

Schlagwörter: Jugendliche, junge Erwachsene, Väter, Geschlecht, Symptombelastung

\begin{abstract}
:
In a longitudinal study, the influence of three types of fathers on their children's psychopathology in adolescence and young adulthood was analyzed. In a sample of 213 subjects, the symptomatology was evaluated at five points in adolescence (Youth Self-Report) and in young adulthood (Young Adult Self-Report). The results show elevated levels of internalizing symptomatology in females compared to males. Furthermore, the results point to significant problems in young adults who have described their father as increasingly negative or distant through adolescence. At all measurement points, these two groups show higher symptomatology than the group of young adults who have described their father as normative in adolescence.
\end{abstract}

Key words: Adolescents, young adults, fathers, gender, symptomatology

\section{Einleitung}

Der Übergang vom Jugend- zum jungen Erwachsenenalter ist aus psychopathologischer Sicht eine entscheidende Schnittstelle von der aus sich ein Rückgang, bzw. eine Verstärkung von psychischen und körperlichen Symptomen ergeben kann. Längsschnittstudien, 
welche die Kontinuität in der Symptombelastung in diesem zeitlichen Rahmen untersucht haben, sind - insbesondere im deutschsprachigen Raum - bisher eher rar (Wittchen/Perkonigg/Lachner/Nelson 1998; Masten/Roisman/Long/Burt/Obradovic/Riley/Boelcke-Stennes/Tellegen 2005; Salmela-Aro/Aunola/Nurmi, 2008; Reef/Diamantopoulou/van Meurs/ Verhulst/van der Ende 2009). Im Übrigen haben sich auch widersprüchliche Befunde ergeben, welche zeigen, dass frühere Symptombelastungen sistieren, während sich andere Symptome neu entwickeln können. Bislang wurde der Rückgang von Symptomen bzw. ein Anstieg von Symptomen vor allem mit den anstehenden Entwicklungsaufgaben im Jugendalter bzw. im jungen Erwachsenenalter in Verbindung gebracht und ins Feld geführt, dass mögliche Anstiege im jungen Erwachsenenalter mit der schwierigen Übergangsphase und den zahlreichen neuen Aufgaben des emerging adulthood (Arnett 2004; SeiffgeKrenke 2006) zusammenhängen. Selten wurde geprüft, inwieweit die Beziehung zu den Eltern langfristige Auswirkungen auf die Symptombelastung ihrer Kinder bis weit ins Erwachsenenalter hinein hat. Gegenstand dieses Artikels ist der Einfluss der Vater-KindBeziehung im Jugendalter auf die langfristige Veränderung der Symptombelastung, eine Perspektive, die trotz der wiederholten Forderung von Phares und Compas (1992) bzw. Phares, Renk, Duhig, Fields und Sly (2009) bislang noch nicht Einzug in die Forschung zur Psychopathologie gefunden hat.

\section{Kontinuität oder Veränderung von Symptombelastung im Jugend- und jungem Erwachsenenalter?}

Die Forschung zur Symptombelastung im Jugendalter ist insbesondere im deutschsprachigen Raum (Döpfner/Plück/Berner/Fegert/Huss/Lenz/Schmeck/Lehmkuhl/Poustka/ Lehmkuhl 1997), aber auch im U.S.-amerikanischen Raum (Achenbach/Howell/McConaughy/Stanger 1995) gut belegt. Längsschnittstudien wie die EDSP-Studie (Early Developmental Stages of Psychopathology, Wittchen et al. 1998) helfen dabei die Ätiologie psychischer Störungen besser zu verstehen. Allerdings hat der Verlauf der internalisierenden und externalisierenden Symptombelastung von der Adoleszenz zum jungen Erwachsenenalter kontroverse Befunde erbracht. Als typisch für die frühe Adoleszenz gelten Belastungen aus den einsetzenden körperlichen Veränderungen der Pubertät und dem gleichzeitig stattfindenden schulischen Belastungen (Sontag/Graber/Clemans 2011). Die Kumulation dieser normativen Stressoren zusammen mit den Implikationen, die sie für die Beziehung zu Eltern und Gleichaltrigen haben, kann eine erhebliche alltägliche Stressbelastung darstellen (Seiffge-Krenke 2011). Bezüglich der Frage, inwiefern sich diese Stressbelastung im Verlauf der Adoleszenz in einer erhöhten Symptombelastung niederschlägt, herrscht allerdings Uneinigkeit in der empirischen Forschung. Insgesamt fanden zahlreiche Studien eine hohe Stabilität der Symptombelastung von der späten Kindheit bis ins mittlere Jugendalter (Sourander/Helstelä 2008; Shin/Sung/Lim/Park/Cho 2012). Während einige Studien allerdings eine Abnahme in der internalisierenden Symptombelastung im Verlauf der Adoleszenz fanden (Sheidow/Strachan/Minden/Henry/Tolan/Gorman-Smith 2008), zeigte sich in anderen Studien eine Zunahme der externalisierenden Auffälligkeiten in dieser Entwicklungsphase (Dekovic/Buist/Reitz 2004; Ham- 
pel/Pössel 2012). Eine hohe Stabilität wird vor allem bei externalisierenden Symptombelastungen gefunden, die spätere Symptombelastung besser vorhersagte als internalisierende Auffälligkeiten (Shin/Sung/Lim/Park/Cho 2012). Weitere Studien fanden einen Wechsel in den relevanten Symptommustern: In der Studie von Bornstein und Kollegen (Bornstein/Hahn/Haynes 2010) sagte die internalisierende Symptombelastung im Alter von 10 Jahren externalisierende Auffälligkeiten im Alter von 14 Jahren vorher. Während einige Autoren einen weiteren Anstieg an internalisierenden Symptomen in der späten Adoleszenz fanden (van Oort/Greaves-Lord/Verhulst/Ormel/Huizink 2009), weisen die Ergebnisse einer Studie von Seiffge-Krenke (2000) darauf hin, dass die Jugendlichen ab dem Alter von 15 Jahren gelernt haben mit den neuartigen Stressoren ohne einen weiteren Anstieg an Symptombelastung umzugehen. Die Studienlage bezüglich des Verlaufs der Symptombelastung im Jugendalter ist folglich widersprüchlich und die Ursachen für die kontroversen Befunde sind unklar. Im vorliegenden Artikel wird daher der Beitrag von Familienbeziehungen, speziell der Beziehung zum Vater, zur Aufklärung möglicher Unterschiede herangezogen.

Die Befundlage zur Symptombelastung im jungen Erwachsenenalter ist ebenfalls widersprüchlich. Zum einen gibt es Studien, die einen Rückgang an psychischen Symptomen und ein gesteigertes Wohlbefinden aufzeigen. So konnten Schulenberg und Zarrett (2006) eine kontinuierliche Zunahme des Wohlbefindens vom 18. bis 26. Lebensjahr feststellen. Auch Galambos und Krahn (2008) wiesen diesen Anstieg nach, bei gleichzeitiger Reduktion depressiver Affekte. Die Angstsymptome (Galambos/Krahn 2008) nehmen ab, und die Prävalenz von Phobien reduziert sich (Tanner/Reinherz/Beardsea/Fitzmaurice/ Leis/Berger 2007). Andererseits wird für die betrachtete Lebensphase das Phänomen der quarterlife crisis beschrieben, dementsprechend empfindet ein Teil der jungen Erwachsenen Ängste in Bezug auf die Herausforderungen und die Instabilität dieses Entwicklungsabschnittes mit seiner erhöhten Exploration im beruflichen und privaten Bereich (Arnett 2004). $\mathrm{Zu}$ bedenken ist auch, dass die Prävalenz der major depression in diesem Lebensabschnitt einen Höhepunkt hat (Schulenberg/Zarrett 2006). Aufschlussreich sind in diesem Zusammenhang Längsschnittstudien wie die von Salmela-Aro, Aunola und Nurmi (2008), in der Universitätsstudenten über einen Zeitraum von 10 Jahren begleitet wurden. $16 \%$ der jungen Erwachsenen wiesen einen hohen und zunehmenden Level an Depressivität auf. Lieb und Kollegen (Lieb/Isensee/Höfler/Pfister/Wittchen 2002) spezifizierten dieses Ergebnis hinsichtlich der elterlichen Psychopathologie an Daten aus der EDSPLängsschnittstudie: Sie wiesen einen stark erhöhten Anstieg in der Inzidenz der major depression im Jugendalter für Kinder depressiver Eltern nach, welcher im jungen Erwachsenenalter seinen Höhepunkt erreicht. Andererseits zeigen Studien der Arbeitsgruppe um Frank Verhulst (Hofstra/van der Ende/Verhulst 2001; Reef et al. 2009), dass nur 22\% der in der Kindheit und Jugend als auffällig klassifizierten Personen auch als junge Erwachsene symptomatisch auffällig wurden. Weitere Studien wie die von Masten und Kollegen (2005) belegen eine hohe Kontinuität der Symptombelastung beim Übergang ins junge Erwachsenenalter. Dies gilt sowohl für internalisierende als auch für externalisierende Auffälligkeiten. Dabei war der Zusammenhang der internalisierenden Auffälligkeiten vor und nach dem Übergang ins Erwachsenenalter für männliche junge Erwachsene im Vergleich zu weiblichen jungen Erwachsenen leicht erhöht. Dies wird von den Autoren mit der höheren interindividuellen Varianz in der Entwicklung junger Frauen begründet (Mas- 
ten et al. 2005). Zusammenfassend zeigen die Befunde, dass im jungen Erwachsenenalter eine hohe Varianz bezüglich der psychischen Gesundheit besteht. Für den Großteil der jungen Erwachsenen zeigt sich eine Verringerung der Symptombelastung, aber auch $\mathrm{Zu}$ nahmen konnten, wenn auch für eine relativ kleine Gruppe, nachgewiesen werden (Schulenberg/Zarrett 2006; Salmela-Aro/Aunola/Nurmi 2008).

Unsere Studie soll prüfen, inwieweit die Beziehung zum Vater während der Jugendzeit, in einer kritischen Transitionsphase, relevant zur Erklärung eines unterschiedlichen Verlaufs (Zunahme oder Rückgang der Symptome) ist. Dabei sind zum einen unterschiedliche Symptomatiken zu bedenken (internalisierend vs. externalisierend), die einen unterschiedlichen Verlauf haben können (Hofstra et al. 2001) und zum anderen Geschlechtsunterschiede, die regelhaft in der Symptombelastung im Jugendalter und jungen Erwachsenenalter auftreten. Weibliche Jugendliche bzw. Frauen geben mehr internalisierende Symptome an, männliche Jugendliche bzw. Männer zeigen dagegen gehäuft externalisierende Symptome. Dieser Geschlechtsunterschied lässt sich erstmalig in der Adoleszenz beobachten und setzt sich im Erwachsenenalter fort. Laut der gender intensification hypothesis (Hill/Lynch 1983) verstärken sich die Geschlechtsunterschiede im frühen Jugendalter mit Einsetzen der Pubertät. Neuere Studien bestätigen allerdings, dass kein Symptombereich als typisch für ein Geschlecht zu bezeichnen ist (Grant/Compas/Thurm/McMahon/Gipson/Campbell/Krochock/ Westerholm, 2006). Die gender intensification hypothesis ist in der jüngeren Vergangenheit ebenfalls kritisch betrachtet worden, da sich in neuen Studien teilweise keine gender intensification in der Adoleszenz mehr gezeigt hat (Priess/ Lindberg/Hyde 2009). Die Autoren erklären dies mit der Angleichung der Geschlechterrollen.

\subsection{Väter und die Symptombelastung ihrer Kinder in Adoleszenz und jungem Erwachsenenalter}

Bereits vor zwei Jahrzehnten hatten Compas und Phares (1992) in ihrem berühmten Aufsatz „The role of fathers in child and adolescent psychopathology: Make room for daddy“ darauf hingewiesen, dass nur in rund $2 \%$ der Studien zur Psychopathologie von Kindern und Jugendlichen untersucht wurde, inwieweit die Väter Einfluss auf die Symptombelastung ihrer Kinder haben. In den letzten Jahren hat allerdings verstärkt Forschung zu Vätern stattgefunden, doch konzentrierte sie sich zunächst auf die negative Seite von Vätern; so finden wir zahlreiche Berichte und Studien über - vor allem sexualisierte - Gewalt ausübende Väter (Finkelhor/Ormrod/Turner 2007; Slep/O'Leary 2005). Dagegen gibt es in den letzten Jahren verstärkt Hinweise auf die „,neuen Väter“, auf positive Emotionen und Zärtlichkeit, d.h. ein anderes Verständnis von Väterlichkeit (Seiffge-Krenke 2012). Die empirische Basis ist schmal, und in vielen Untersuchungen wird unausgesprochen angenommen, dass sich Väter verhalten müssen wie Mütter (Seiffge-Krenke 2001). Damit deutet sich an, dass offenkundig auch gegenwärtig sehr verschiedene Typen von Vätern existieren, die möglicherweise auch einen unterschiedlichen Einfluss auf die Symptombelastung ihrer Kinder haben können.

Ganz generell hat die Forschung an Vätern in der Entwicklungspsychologie gezeigt, dass Väter, verglichen mit Müttern (Seiffge-Krenke 2001), einerseits eine distanziertere Position zu ihren Kindern einnehmen und dass sie zugleich andere Funktionen für ihre 
Kinder haben als Mütter. Diese distinktiven Funktionen des Vaters umfassen die Unterstützung des Explorationsverhaltens und die Autonomieförderung (Shulman/SeiffgeKrenke 1997) sowie die Unterstützung der Berufsfindung (Cabrera/Tamis-LeMonda/ Bradley/\&Hofferth/Lamb 2000). Gerade die Förderung der Autonomie des Kindes wird zu großen Teilen durch den Vater betrieben (Shulman/Seiffge-Krenke 1997). Die Jugendlichen in der Studie von Shulman und Kollegen (2009) fühlten ihr Bestreben nach Unabhängigkeit durch ihren Vater besser verstanden und respektiert als durch ihre Mutter (Shulman/Kalnitzki/Shahar 2009). Für den bevorstehenden Übergang ins Erwachsenenalter ist der Vater nach wie vor von essentieller Bedeutung für sein Kind. Shulman und Kollegen (2009) sprechen in diesem Zusammenhang, unter Bezugnahme auf die einerseits warme, aber durchaus auch distanzierte Erziehung des Vaters von einem „close enough parent" (Shulman/Kalnitzki/Shahar 2009: 259). In dieser Rolle lässt der Vater der Individuation seines erwachsenen Kindes besonders viel Raum und unterstützt es dabei, geht dabei aber auch realistischer mit den Problemen des jungen Erwachsenen um als die Mutter (Shulman/Noy-Sever 2006).

Die wenigen Studien, die die Symptombelastung von Kindern im Zusammenhang mit der Beziehung zu ihrem Vater analysiert haben, stellten ganz generell fest, dass eine unterstützende Vater-Kind-Beziehung ein protektiver Faktor ist. Amato und Rivera (1999) fanden beispielsweise einen negativen Zusammenhang zwischen väterlicher Involviertheit und Verhaltensauffälligkeiten des Kindes. Eine hohe Involviertheit von Seiten des Vaters hängt dementsprechend mit einer geringen Anzahl von Verhaltensauffälligkeiten seines Kindes zusammen. Diese Erkentnisse wurden in der längsschnittlich angelegten Studie von Flouri und Buchanan (2003) bestätigt: Väterliche Involviertheit in der Kindheit wirkte hier als protektiver Faktor bei emotionalem Stress und Entwicklungsabweichungen im Jugendalter. Auch väterliche Verhaltenskontrolle, in einem angemessenen Umfang, scheint sich positiv auf die Symptombelastung auszuwirken und den Anstieg von externalisierenden Symptomen deutlich zu bremsen (Galambos/Barker/Almeida 2003; Stolz/Barber/Olsen 2005). Auch die wahrgenommene Nähe zum Vater war in der Studie von Booth, Scott und King (2010) von kritischer Bedeutung, da Jugendliche, die eine geringe Nähe zu ihrem Vater angaben, eher delinquentes Verhalten zeigten (Booth/Scott/King 2010). Andererseits ist zu bedenken, dass in dieser Transitionsphase vom Jugendalter zum Erwachsenenalter Kinder auch erhebliche Konflikte mit ihren Vätern haben können. Unterstützung, wahrgenommene Verfügbarkeit, aber auch Distanz und Konfliktbelastung sind demnach Dimensionen in der Vater-Kind-Beziehung, die von zentraler Bedeutung für jugendliche und erwachsene Kinder sind. Ungeklärt sind auch die Auswirkungen des wahrgenommenen Vatertyps auf die Symptombelastung im Jugendalter und jungen Erwachsenenalter in Abhängigkeit von familienstrukturellen Veränderungen. Dies gilt sowohl für Zwei-Eltern-Haushalte, als auch für Patchworkfamilien sowie Haushalte mit alleinerziehendem Vater; einer Familienform, welche in Deutschland immer mehr an Bedeutung gewinnt (Matzner 2007). Wegen der hohen Anzahl von Patienten aus Trennungs- und Scheidungsfamilien in klinischen Stichproben ist dies eine vordringliche Aufgabe. Deshalb wurde von uns ebenfalls geprüft, inwiefern sich Jugendliche, welche bei einem alleinerziehenden Elternteil aufwachsen in der Symptombelastung von Jugendlichen aus einem Zwei-Eltern-Haushalt unterscheiden.

Der vorliegende Artikel baut auf einer früheren Forschungsarbeit von Seiffge-Krenke, Overbeek und Vermulst im Jahr 2010 auf, die drei unterschiedlich wahrgenommene Väter- 
typen bei Jugendlichen über einen Zeitraum von mehreren Jahren ermittelt hatte: Der normative Vatertypus wird als unterstützend wahrgenommen und ist mit positiven Affekten besetzt. Dieser Vatertypus wird als unterstützend empfunden. Der distanzierte Vater zeichnet sich in der Wahrnehmung der Kinder durch Unnahbarkeit und Unerreichbarkeit aus. Dabei muss nicht unbedingt eine räumliche Trennung, bzw. Scheidung der Eltern vorliegen; die Jugendlichen erlebten ihren Vater auch in intakten Familien als wenig emotional verfügbar. Sie hatten nicht das Gefühl, Unterstützung beim Vater suchen zu können, berichteten aber auch nicht über viele Konflikte oder starke negative Emotionen gegenüber ihrem Vater. Der negative Vatertypus basiert auf der Einschätzung Jugendlicher, die sich durch ihren Vater nicht angemessen unterstützt fühlen. Diese Jugendlichen beschreiben die Beziehung zu ihrem Vater als konfliktreich und ihren Vater zunehmend als negativ.

Diese Ergebnisse bilden die Grundlage für die vorliegende Studie, in der die Auswirkungen auf die Symptombelastung über einen Zeitraum von 9 Jahren untersucht wurden.

\section{Hypothesen und Fragestellung}

Im Folgenden werden die erwarteten Unterschiede bezüglich des Verlaufs in der Symptombelastung dargestellt. Dabei werden sowohl das Geschlecht als auch die erwarteten Auswirkungen des wahrgenommenen Vatertyps berücksichtigt.

In Einklang mit bisheriger Forschung erwarten wir vermehrt internalisierende Symptombelastung bei den weiblichen, und externalisierende Symptombelastung bei den männlichen Probanden. Wir gehen im Sinne der gender intensification hypothesis (Hill/ Lynch 1983) davon aus, dass sich zu Beginn der Adoleszenz Geschlechtsunterschiede zeigen, welche im jungen Erwachsenenalter wieder abnehmen werden. Es wird des Weiteren erwartet, dass Jugendliche mit einem alleinerziehenden Elternteil höhere externalisierende Symptombelastung aufweisen.

Basierend auf der Unterscheidung in drei verschiedene Vatertypen (Seiffge-Krenke et al. 2010) wird hinsichtlich der Symptombelastung im Jugendalter davon ausgegangen, dass die Symptombelastung in der Gruppe der Jugendlichen, welche ihren Vater als normativ beschreibt, mit dem Übergang ins junge Erwachsenenalter absinkt, da die unterstützende väterliche Beziehung als ein Puffer gegen Stressbelastung des Kindes im Übergang funktionieren könnte. Ein ähnlicher Verlauf wird auch in der Gruppe der Jugendlichen, welche ihren Vater als distanziert beschreiben, erwartet; allerdings erwarten wir hier einen weniger starken Rückgang der Symptombelastung. Für die Gruppe der Jugendlichen, welche ihren Vater als negativ beschreiben, sind hingegen durchgehend hohe Werte in der Symptombelastung sowohl im Jugend- als auch im jungen Erwachsenalter der Kinder zu vermuten. Diese Gruppe zeichnet sich durch stark negative Affekte gegenüber dem eigenen Vater aus, was möglicherweise zu vermehrten Konflikten und Stress und damit zu einer zunehmenden Symptombelastung führen kann. 


\section{Methode}

\subsection{Stichprobe}

In der vorliegenden Studie wurde eine Stichprobe untersucht, zu der bereits Daten aus ihrer Jugendzeit vorlagen. Die Einteilung der Vatertypen, d.h. die Einschätzung des väterlichen Verhaltens durch die Jugendlichen, basiert auf der bereits erwähnten Studie von Seiffge-Krenke et al. (2010). In dieser früheren Studie wurde eine Stichprobe Jugendlicher mit dem NRI (Network of Relationships Inventory, Furman/Buhrmester, 1985), der soziale Unterstützung zu beiden Eltern erfasst, untersucht. Auf der Basis des Verlaufs über 4 Jahre wurden anschließend Wachstumsmodelle mit dem Programm Mplus (Muthén/ Muthén 2006) berechnet. Die Autoren teilten die Stichprobe auf der Basis der Einschätzung der Jugendlichen in den beiden Dimensionen support-closeness (Unterstützung-Nähe) und negative affect (negativer Affekt) bezogen auf ihren Vater in drei Gruppen von Entwicklungsverläufen ein: Jugendliche, welche ihren Vater über diesen Zeitverlauf als normativ beschreiben, geben durchgehend mittlere Werte in den beiden Dimensionen Unterstützung-Nähe und negativer Affekt an; Jugendliche, welche ihren Vater als distanziert beschreiben, geben geringere Werte in Unterstützung-Nähe und mittlere Werte in negativem Affekt an; Jugendliche, welche ihren Vater als negativ beschreiben, geben geringe Werte in Unterstützung-Nähe und über die Zeit steigende Werte in negativem Affekt an. Um eine Passung der Daten zu gewährleisten wurde das Bayesianische Informationskriterium (BIC) verwendet (Seiffge-Krenke et al. 2010).

Die Stichprobe der 213 Probanden (104 männlich, 109 weiblich) wurde im Jugendsowie im jungen Erwachsenenalter untersucht. Sie stellen einen repräsentativen Querschnitt der deutschen Bevölkerung bezüglich der Variablen Alter, Geschlecht und sozioökonomischer Status (SES) dar. Die Probanden waren zum Zeitpunkt der ersten Befragung 14 Jahre $(\mathrm{M}=13.88, \mathrm{SD}=1.33)$ und zum letzten Messzeitpunkt 23 Jahre alt $(\mathrm{M}=$ 22.96, $\mathrm{SD}=1.44$ ). Der Dropout über die gesamte Untersuchung lag bei $35.96 \%$.

Tabelle 1: Beschreibung der soziodemographischen Kennwerte der Stichprobe zum letzten Messzeitpunkt (23 Jahre) gesamt und nach den drei Vatertypen

\begin{tabular}{|c|c|c|c|c|c|c|c|c|c|c|c|}
\hline & & \multicolumn{2}{|c|}{ Gesamt } & \multicolumn{2}{|c|}{$\begin{array}{c}\text { Vater } \\
\text { normativ }\end{array}$} & \multicolumn{2}{|c|}{$\begin{array}{c}\text { Vater } \\
\text { distanziert }\end{array}$} & \multicolumn{2}{|c|}{$\begin{array}{c}\text { Vater } \\
\text { negativ }\end{array}$} & $\begin{array}{l}\text { F/Chi- } \\
\text { Quadrat }\end{array}$ & $p$ \\
\hline Anzahl & $N$ & \multicolumn{2}{|c|}{213} & \multicolumn{2}{|c|}{174} & \multicolumn{2}{|c|}{11} & \multicolumn{2}{|c|}{28} & & \\
\hline Alter & $\begin{array}{l}M \\
S D\end{array}$ & \multicolumn{2}{|c|}{$\begin{array}{c}22.96 \\
1.44\end{array}$} & \multicolumn{2}{|c|}{$\begin{array}{c}22.91 \\
1.46\end{array}$} & \multicolumn{2}{|c|}{$\begin{array}{c}23.67 \\
1.21\end{array}$} & \multicolumn{2}{|c|}{$\begin{array}{c}23.00 \\
1.41 \\
\end{array}$} & 0.78 & .460 \\
\hline Häufigkeiten* & & $f$ & $f \%$ & $f$ & $f \%$ & $f$ & $f \%$ & $f$ & $f \%$ & \multirow{3}{*}{.545} & \multirow{3}{*}{.761} \\
\hline \multirow{2}{*}{ Geschlecht } & $M$ & 104 & 48.8 & 87 & 50.0 & 5 & 45.5 & 12 & 42.9 & & \\
\hline & $W$ & 109 & 51.2 & 87 & 50.0 & 6 & 54.5 & 16 & 57.1 & & \\
\hline \multirow{4}{*}{ Familienstruktur } & Verheiratet & 176 & 83.8 & 152 & 88.4 & 2 & 18.2 & 22 & 81.5 & \multirow{4}{*}{46.51} & \multirow{4}{*}{$<.001$} \\
\hline & Eheähnlich & 4 & 1.9 & 3 & 1.7 & 1 & 9.1 & 0 & 0.0 & & \\
\hline & Alleinerziehend & 22 & 10.5 & 13 & 7.6 & 7 & 63.6 & 2 & 7.4 & & \\
\hline & Sonstiges & 8 & 3.8 & 4 & 2.3 & 1 & 9.1 & 3 & 11.1 & & \\
\hline \multirow{4}{*}{ SES } & Hoch & 78 & 36.6 & 64 & 36.8 & 5 & 45.5 & 9 & 32.1 & \multirow{4}{*}{10.46} & \multirow{4}{*}{.106} \\
\hline & Mittel & 103 & 48.4 & 84 & 48.3 & 2 & 18.2 & 17 & 60.7 & & \\
\hline & Niedrig & 28 & 13.1 & 23 & 13.2 & 4 & 36.4 & 1 & 3.6 & & \\
\hline & Keine Aussage & 4 & 1.9 & 3 & 1.7 & 0 & 0.0 & 1 & 3.6 & & \\
\hline
\end{tabular}

* $\mathrm{f}=$ absolute Häufigkeiten; $\mathrm{f} \%$ = relative Häufigkeiten (in \%) 
Die Angaben bezüglich der soziodemographischen Variablen wurden zu allen Messzeitpunkten erhoben und sind im Wesentlichen gleich geblieben. Tabelle 1 gibt die Messwerte zum letzten Messzeitpunkt wieder, und stellt die Soziodemographie der Gesamtstichprobe und die drei untersuchten Gruppen mit verschieden wahrgenommenen Vatertypen (Vater normativ, Vater distanziert, Vater negativ) dar.

Wie der Tabelle $1 \mathrm{zu}$ entnehmen ist, zeigen sich bezüglich des SES, des Geschlechts und des Alters keine signifikanten Unterschiede innerhalb der Stichprobe. Die Familienstruktur ist unterschiedlich mit einem höheren Anteil alleinerziehender Elternteile in der Gruppe der Jugendlichen, welche ihren Vater im Jugendalter als distanziert beschrieben haben.

\subsection{Messinstrumente}

In der vorliegenden Untersuchung wurde der Youth Self Report und der Young Adult Self Report (beide Achenbach 1997) benutzt. Beide Instrumente werden im Folgenden dargestellt.

Die Symptombelastung der Jugendlichen wurde jährlich anhand des Youth Self Report (YSR) von Achenbach (1997) im Alter von 14 bis 17 Jahren erfragt. Im jungen Erwachsenenalter (im Alter von 23 Jahren) wurde der Young Adult Self Report (YASR, Achenbach 1997)) benutzt, der die Adaption des YSR für junge Erwachsene darstellt. Der YSR/ YASR dient als Screeningverfahren der Erfassung klinisch relevanter internalisierender und externalisierender Auffälligkeiten. Der zweite Abschnitt des YSR/YASR besteht aus einer Liste mit 130 Symptomen. Die Aussagen bezüglich der 119 Items des YSR/YASR sind auf einer dreistufigen Skala (Range 0 bis 2, wobei $0=$ „nicht zutreffend“, 1 = „trifft etwas/manchmal zu“, 2 = ,trifft genau/häufig zu“) für die vergangenen sechs Monate einzuschätzen. Wir verwendeten eine gekürzte Fassung des YSR/YASR mit 24 Items. Es wurden die beiden Faktoren zweiter Ordnung, internalisierende und externalisierende Störungen sowie ein Gesamtsymptombelastungswert berechnet. Der Faktor internalisierende Störungen wurde anhand von 14 Items mit den Subskalen Ängstlichkeit/Depressivität (5 Items, Beispielitem: „Ich fühle mich einsam“), sozialer Rückzug (5 Items, Beispielitem: „Ich bin sehr schweigsam“) und körperliche Beschwerden (4 Items, Beispielitem: „Ich schlafe öfter oder länger als andere“) erfasst. Die interne Konsistenz der internalisierenden Störungen beträgt $\alpha=.82$.

Der Faktor externalisierende Störungen wurde mit 10 Items erfasst und umfasst die Subskalen: Aggressives Verhalten (4 Items, Beispielitem: "Ich streite viel") und delinquente Verhaltensweisen (6 Items, Beispielitem: „Ich lüge oder betrüge oft"). Die interne Konsistenz der externalisierenden Störungen beträgt $\alpha=.58$.

\subsection{Statistische Auswertung}

Die Daten wurden mit dem Datenverarbeitungsprogramm SPSS (Statistical Program for the Social Sciences 18.0) ausgewertet. Der in Längsschnittstudien übliche Dropout wurde durch eine Missing-Data-Korrektur korrigiert. Dafür wurde die Methode der multiplen 
Imputation im Datenverarbeitungsprogramm SPSS (Statistical Program for the Social Sciences 18.0) durchgeführt. Als Einflussvariablen wurden Geschlecht, Alter, sozioökonomischer Status und die Gruppierungsvariable Vatertypen verwendet. Nach Durchführen der multiplen Imputation wurden die Werte anhand univariater Varianzanalysen mit den Ausgangswerten verglichen. Bei Werten von $p>.01$ wurde eine erneute multiple Imputation durchgeführt.

Nach der Durchführung der Missing-Data-Korrektur wurden die Daten zur Symptombelastung aus dem Jugend- und jungen Erwachsenenalter der Probanden anhand von univariaten Varianzanalysen mit Messwiederholung über die Gruppen mit im Jugendalter unterschiedlich wahrgenommenen Vatertypen verglichen. Dabei wurden die Verläufe der Symptombelastung über die Zeit (für Geschlecht, Familienstand der Eltern und die Gruppierungsvariable Vatertypen) anhand dreier univariater Varianzanalysen mit Messwiederholung analysiert. Die internalisierenden und externalisierenden Auffälligkeiten sowie die Gesamtauffälligkeiten wurden hierbei separat untersucht.

\section{Ergebnisse}

\subsection{Der Verlauf der Symptombelastung von der Jugendzeit zum jungen Erwachsenenalter insgesamt und im Geschlechtsvergleich}

Bei der Analyse des Verlaufs der internalisierenden und externalisierenden Auffälligkeiten sowie der Gesamtauffälligkeiten zeigt sich ein Rückgang der internalisierenden Symptombelastung von der ersten Erhebung im Alter von 14 Jahren auf die folgenden Zeitpunkte $\left(\mathrm{F}(4,223)=6.73 ; \mathrm{p}<.001 ; \eta^{2}=.108\right)$. Die externalisierende Symptombelastung zeigt ebenfalls einen Abfall von den ersten beiden Zeitpunkten im Alter von 14 und 15 Jahren auf den Messzeitpunkt im Alter von 17 Jahren. Des Weiteren fällt die externalisierende Symptombelastung mit 17 Jahren auf die externalisierende Symptombelastung mit 23 Jahren signifikant ab $\left(\mathrm{F}(4,223)=37.21 ; \mathrm{p}<.001 ; \eta^{2}=.400\right)$. Die Gesamtsymptombelastung fällt vom ersten Erhebungszeitpunkt mit 14 Jahren signifikant auf die Erhebungszeitpunkte mit 15, 16 und 17 Jahren ab und steigt dann zum jungen Erwachsenenalter mit 23 Jahren hin wieder an $\left(\mathrm{F}(4,223)=25.23 ; \mathrm{p}<.001 ; \eta^{2}=.312\right)$ 
Abbildung 1: Verlauf der internalisierenden Auffälligkeiten in der Gesamtstichprobe und nach Geschlecht getrennt

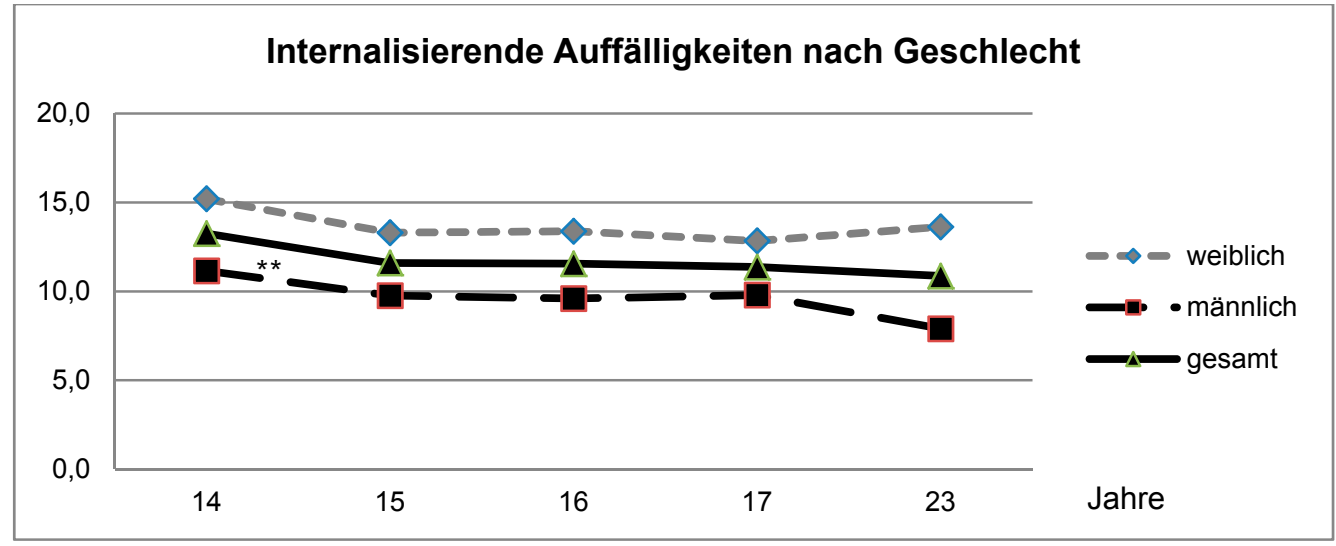

$* p<.05 ; * * p<.01$

Abbildung 2: Verlauf der externalisierenden Auffälligkeiten in der Gesamtstichprobe und nach Geschlecht getrennt

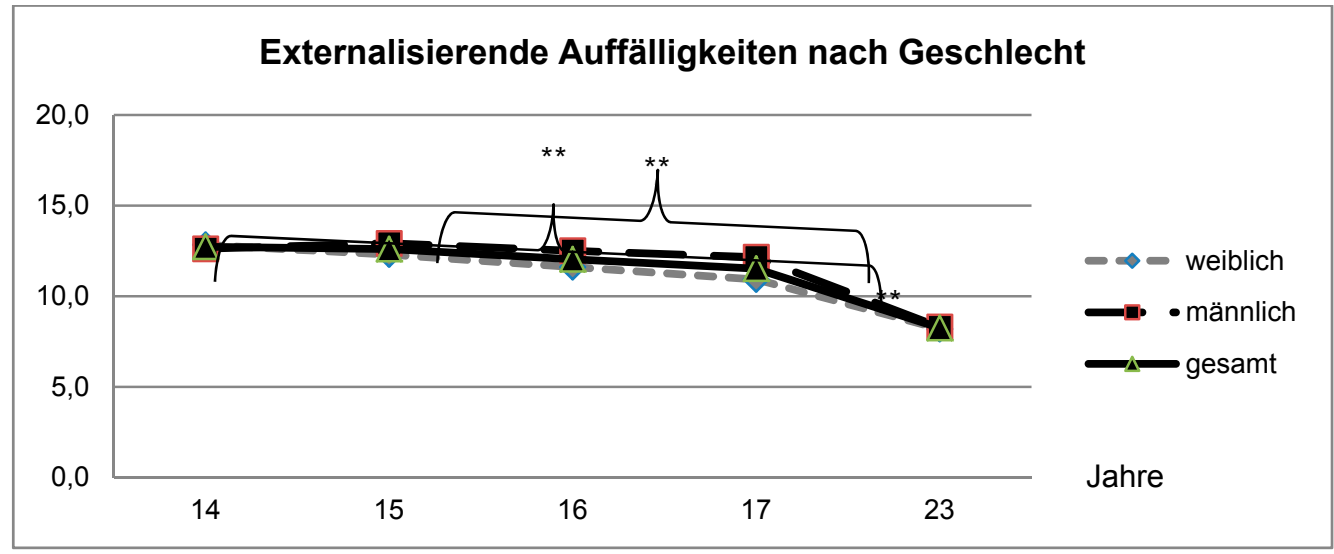

$* p<.05 ; * * p<.01$

Im Geschlechtsvergleich zeigt sich, dass die weiblichen im Vergleich zu den männlichen Probanden zu jedem Messzeitpunkt signifikant höhere Werte in der internalisierenden Symptombelastung aufweisen: Dies ist im Alter von $14\left(\mathrm{~F}(1,226)=17.36 ; \mathrm{p}<.001 ; \eta^{2}=\right.$ $.071), 15\left(\mathrm{~F}(1,226)=12.94 ; \mathrm{p}<.001 ; \eta^{2}=.054\right), 16\left(\mathrm{~F}(1,226)=13.27 ; \mathrm{p}<.001 ; \eta^{2}=\right.$ $.055), 17\left(\mathrm{~F}(1,226)=9.29 ; \mathrm{p}=.003 ; \eta^{2}=.039\right)$ und 23 Jahren $(\mathrm{F}(1,226)=28.98 ; \mathrm{p}<$ $\left..001 ; \eta^{2}=.114\right)$ der Fall. Die weiblichen geben im Vergleich zu den männlichen Probanden außerdem höhere Gesamtauffälligkeiten mit $14\left(\mathrm{~F}(1,226)=8.43 ; \mathrm{p}=.004 ; \eta^{2}=.036\right)$ und 23 Jahren $\left(F(1,226)=15.92 ; \mathrm{p}<.001 ; \eta^{2}=.066\right)$ an. 
Abbildung 3: Verlauf der Gesamtauffälligkeiten in der Gesamtstichprobe und nach Geschlecht getrennt

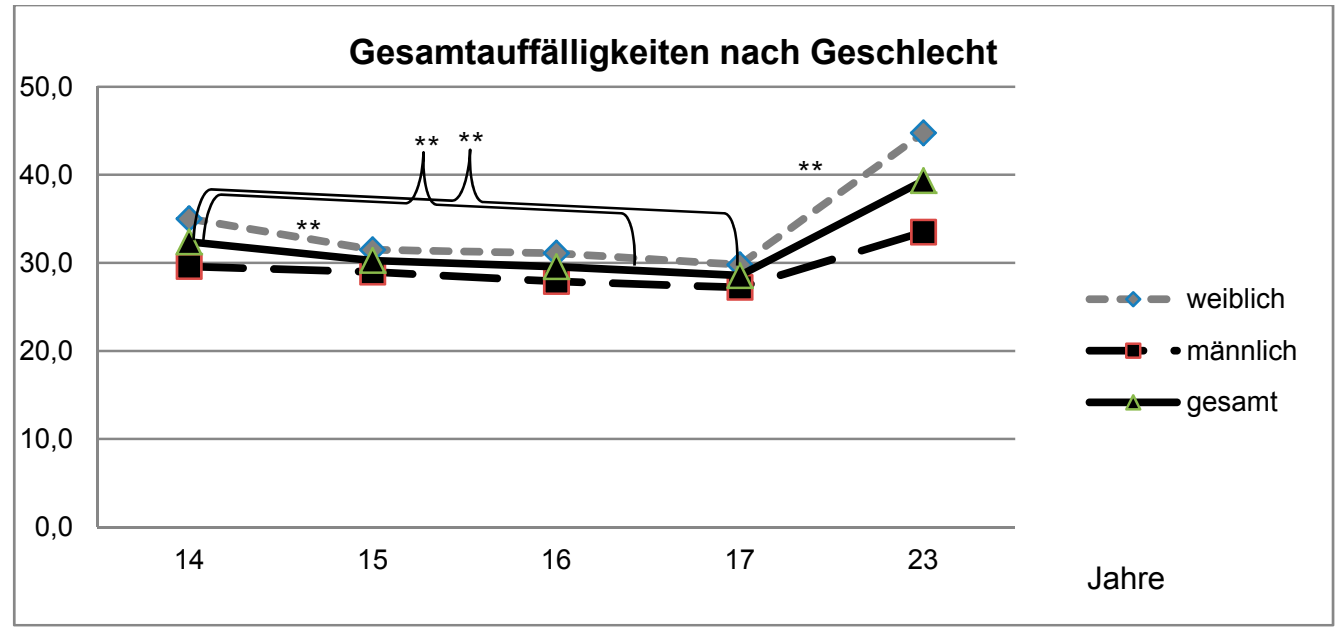

$* p<.05 ; * * p<.01$

Tabelle 2: Die Symptombelastung in Jugendalter und jungem Erwachsenenalter im Vergleich über das Geschlecht

\begin{tabular}{|c|c|c|c|c|c|c|c|c|c|}
\hline \multirow{3}{*}{$\begin{array}{l}\text { Skala (Alter) } \\
\text { Externalisierende Auffälligkeiten (14) }\end{array}$} & \multirow{2}{*}{\multicolumn{2}{|c|}{$\begin{array}{l}\text { Gesamt } \\
(\mathrm{N}=228)\end{array}$}} & \multicolumn{2}{|c|}{ Weiblich } & \multicolumn{2}{|c|}{ Männlich } & \multirow{3}{*}{$\begin{array}{c}\mathbf{F} \\
0.07\end{array}$} & \multirow{3}{*}{$\begin{array}{c}\boldsymbol{p} \\
\\
.785\end{array}$} & \multirow{3}{*}{$\begin{array}{c}\eta^{2} \\
.000\end{array}$} \\
\hline & & & \multicolumn{2}{|c|}{$(\mathrm{N}=118)$} & \multicolumn{2}{|c|}{$(\mathrm{N}=110)$} & & & \\
\hline & 12.73 & 5.77 & 12.83 & 5.85 & 12.62 & 5.70 & & & \\
\hline Internalisierende Auffälligkeiten (14) & 13.25 & 7.59 & 15.20 & 8.31 & 11.15 & 6.10 & 17.36 & .000 & .071 \\
\hline Gesamtauffälligkeiten (14) & 32.39 & 14.35 & 35.01 & 16.06 & 29.57 & 11.70 & 8.43 & .004 & .036 \\
\hline Externalisierende Auffälligkeiten (15) & 12.60 & 6.26 & 12.30 & 6.20 & 12.92 & 6.33 & 0.55 & .457 & .002 \\
\hline Internalisierende Auffälligkeiten (15) & 11.59 & 7.61 & 13.30 & 8.52 & 9.76 & 6.02 & 12.94 & .000 & .054 \\
\hline Gesamtauffälligkeiten (15) & 30.26 & 15.03 & 31.48 & 16.68 & 28.95 & 12.97 & 1.62 & .205 & .007 \\
\hline Externalisierende Auffälligkeiten (16) & 12.04 & 6.29 & 11.60 & 6.43 & 12.51 & 6.12 & 1.18 & .279 & .005 \\
\hline Internalisierende Auffälligkeiten (16) & 11.55 & 8.05 & 13.38 & 8.62 & 9.59 & 6.90 & 13.27 & .000 & .055 \\
\hline Gesamtauffälligkeiten (16) & 29.55 & 15.19 & 31.10 & 16.74 & 27.89 & 13.21 & 2.55 & .112 & .011 \\
\hline Externalisierende Auffälligkeiten (17) & 11.52 & 6.60 & 10.93 & 7.00 & 12.15 & 6.10 & 1.97 & .162 & .009 \\
\hline Internalisierende Auffälligkeiten (17) & 11.36 & 7.68 & 12.83 & 8.66 & 9.79 & 6.12 & 9.29 & .003 & .039 \\
\hline Gesamtauffälligkeiten (17) & 28.54 & 15.35 & 29.78 & 17.49 & 27.21 & 12.61 & 1.60 & .207 & .007 \\
\hline Externalisierende Auffälligkeiten (23) & 8.25 & 5.50 & 8.19 & 6.05 & 8.30 & 4.88 & 0.02 & .881 & .000 \\
\hline Internalisierende Auffälligkeiten (23) & 10.86 & 8.51 & 13.62 & 9.06 & 7.90 & 6.74 & 28.98 & .000 & .114 \\
\hline Gesamtauffälligkeiten (23) & 39.32 & 21.97 & 44.75 & 23.15 & 33.50 & 19.08 & 15.92 & .000 & .066 \\
\hline
\end{tabular}

Eine signifikante Interaktion zwischen Geschlecht und Zeit ergab sich nur für die Gesamtsymptombelastung $\left(\mathrm{F}(4,223)=4.99 ; \mathrm{p}=.001 ; \eta^{2}=.082\right)$. 


\subsection{Unterschiede in der Symptombelastung nach dem Familienstand der Eltern}

Im Folgenden werden die Ergebnisse aus der Analyse des Vergleichs der Symptombelastung nach dem Familienstand dargestellt. Hierbei zeigte sich lediglich ein signifikanter Unterschied im Alter von 23 Jahren in der externalisierenden Symptombelastung ( $F$ $\left.(3,220)=3.26 ; p=.022 ; \eta^{2}=.043\right)$. Hier wiesen junge Erwachsene mit einem alleinerziehenden Elternteil höhere Werte auf als junge Erwachsene mit zwei verheirateten Elternteilen.

\subsection{Probanden mit drei verschieden wahrgenommenen Typen von Vätern: Wie verändert sich ihre Symptombelastung von der Jugendzeit zum jungen Erwachsenenalter?}

An dieser Stelle werden die Unterschiede über die Zeit für die Gesamtsymptombelastung sowie für die internalisierenden und externalisierenden Auffälligkeiten separat für die drei Gruppen mit verschieden wahrgenommenen Vatertypen berichtet. Anschließend werden die Unterschiede zwischen den drei Gruppen zu den verschiedenen Messzeitpunkten aufgezeigt (siehe 4.4).

Bei der Analyse des Verlaufs der internalisierenden, externalisierenden und Gesamtauffälligkeiten in den drei Gruppen mit verschieden wahrgenommenen Vatertypen zeigt sich ein gruppenspezifischer Verlauf. In der Gruppe der Probanden, die die Beziehung zu ihrem Vater während ihrer Jugendzeit als ausgeglichen erlebten (normativer Vatertypus), zeigen sich deutliche Reduktionen der Symptombelastungen über das Jugendalter hinweg: Es zeigte sich ein signifikanter Abfall $\left(\mathrm{F}(4,632)=5.32 ; \mathrm{p}<.001 ; \eta^{2}=.030\right)$ der internalisierenden Auffälligkeiten mit 14 Jahren auf die drei Messzeitpunkte im Alter von 15, 16 und 17 Jahren. In den externalisierenden Auffälligkeiten zeigt sich ein signifikanter Abfall $\left(F(4,632)=48.89 ; p<.001 ; \eta^{2}=.220\right)$ vom Messzeitpunkt mit 14 Jahren auf den mit 17 Jahren sowie ein signifikanter Abfall von den ersten vier Messzeitpunkten im Jugendalter auf den letzten Messzeitpunkt im jungen Erwachsenenalter. Die Gesamtauffälligkeiten fallen vom ersten Messzeitpunkt mit 14 Jahren signifikant ab $(\mathrm{F}(4,632)=27.05 ; \mathrm{p}<$ $.001 ; \eta^{2}=.135$ ), verharren dann im Alter von 15 bis 17 Jahren und steigen zum letzten Messzeitpunkt mit 23 Jahren signifikant an.

In der Gruppe der Probanden, welche ihren Vater während der Jugendzeit als distanziert beschreiben, zeigt sich ein signifikanter Abfall in den internalisierenden Auffälligkeiten $\left(F(4,40)=5.21 ; p=.002 ; \eta^{2}=.342\right)$. In den Post-hoc-Tests zeigt sich dieser Effekt in einem tendenziellen Abfall von Messzeitpunkt im Alter von 17 Jahren auf den letzten Messzeitpunkt im Alter von 23 Jahren.

In der Gruppe der Probanden, welche ihren Vater als negativ beschreiben, zeigt sich ein signifikanter Abfall $\left(F(4,108)=3.55 ; p=.009 ; \eta^{2}=.116\right)$ der externalisierenden Auffälligkeiten von Messzeitpunkt im Alter von 15 Jahren auf den letzten Messzeitpunkt im Alter von 23 Jahren. Für die Gesamtauffälligkeiten zeigt sich ein signifikanter Abfall (F $\left.(4,108)=13.57 ; \mathrm{p}<.001 ; \eta^{2}=.335\right)$ von den ersten vier Messzeitpunkten im Jugendalter auf den letzten Messzeitpunkt im Alter von 23 Jahren. Der Verlauf der internalisierenden, 
externalisierenden und der Gesamtauffälligkeiten ist nach Gruppen getrennt in den folgenden drei Abbildungen (Abbildungen 4, 5 und 6) dargestellt.

Abbildung 4: Verlauf der internalisierenden Auffälligkeiten in Gruppen mit verschiedenen Vatertypen

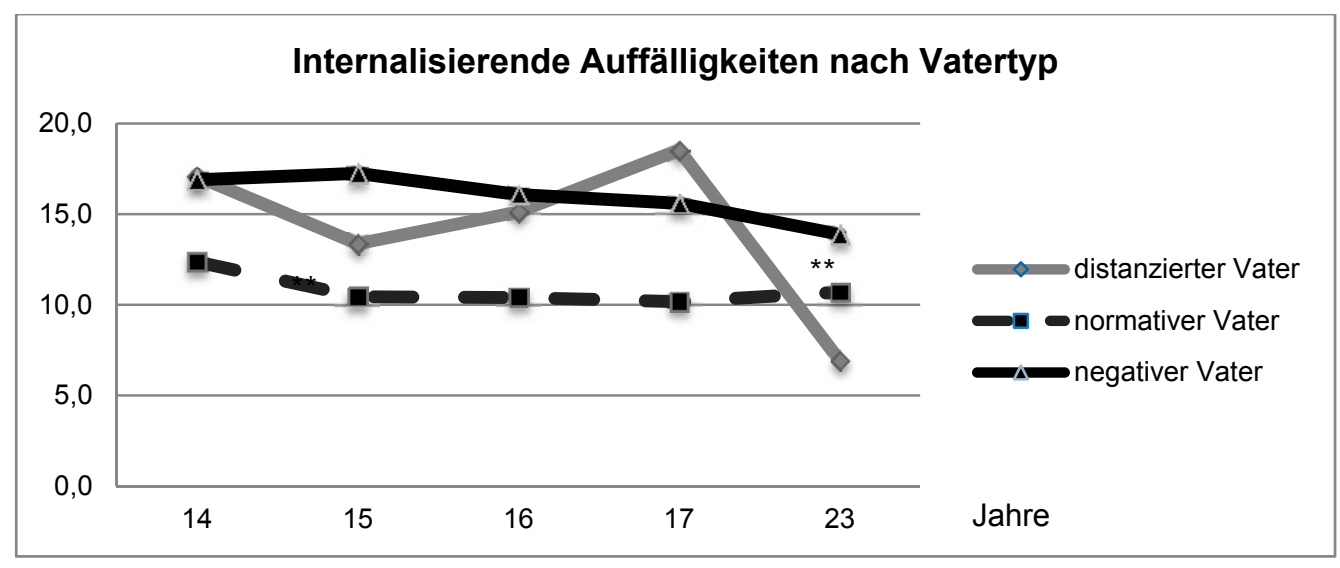

$* p<.05 ; * * p<.01$

Abbildung 5: Verlauf der externalisierenden Auffälligkeiten in Gruppen mit verschiedenen Vatertypen

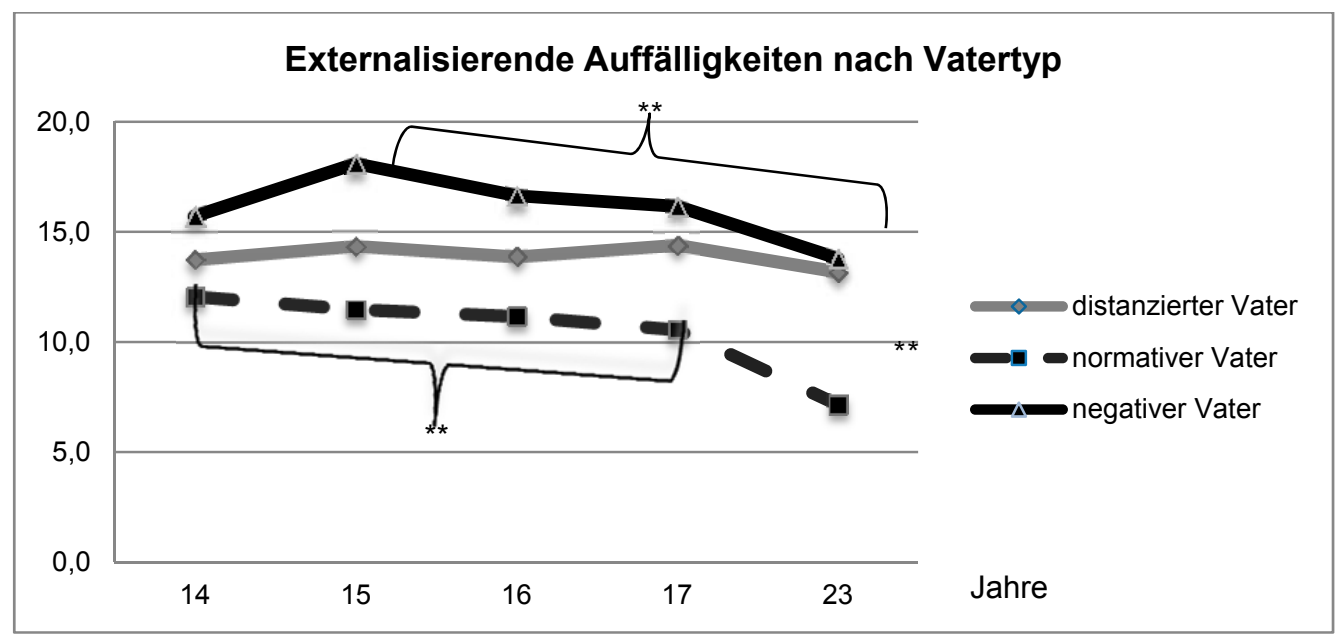

$* p<.05 ; * * p<.01$ 
Abbildung 6: Verlauf der Gesamtauffälligkeiten in Gruppen mit verschiedenen Vatertypen

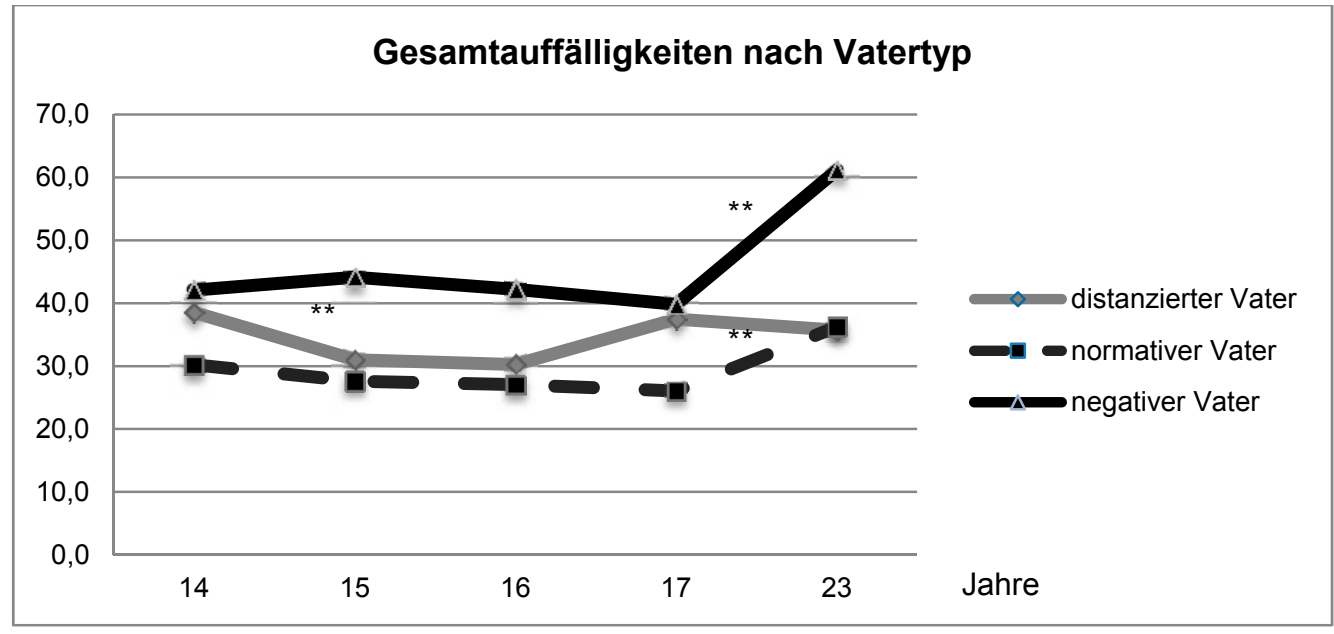

$* p<.05 ; * * p<.01$

\subsection{Unterschiede in der Symptombelastung zwischen den drei Gruppen mit im Jugendalter unterschiedlich wahrgenommenen Vatertypen}

Beim Vergleich der Symptombelastung zeigt sich, dass die Gruppe der Jugendlichen, welche ihren Vater als negativ beschreiben, sich durchgehend signifikant von der Gruppe der Jugendlichen, welche ihren Vater als normativ beschreiben, unterscheidet. Die einzige Ausnahme bildet hier die Skala internalisierende Auffälligkeiten im Alter von 23 Jahren. Es zeigt sich ein Unterschied in den Gesamtauffälligkeiten im Alter von 23 Jahren zwischen der Gruppe der Jugendlichen, welche ihren Vater als negativ beschreiben, und der Gruppe der Jugendlichen, welche ihren Vater als normativ beschreiben $(F(4,113)=$ $18.80 ; \mathrm{p}<.001 ; \eta^{2}=.152$ ). Die Gruppe der Jugendlichen, welche ihren Vater als distanziert beschreiben, unterscheidet sich von der Gruppe der Jugendlichen, welche ihren Vater als normativ beschreiben in der Skala internalisierende Auffälligkeiten im Alter von 17 Jahren $\left(F(4,113)=12.10 ; p<.001 ; \eta^{2}=.103\right)$ und in den Gesamtauffälligkeiten ebenfalls im Alter von 17 Jahren $\left(F(4,113)=13.48 ; p<.001 ; \eta^{2}=.114\right)$. Des Weiteren zeigen die jungen Erwachsenen, welche ihren Vater im Jugendalter als distanziert beschrieben haben, signifikant höhere Werte in der Skala externalisierende Auffälligkeiten im Alter von 23 Jahren $\left(F(4,113)=28.03 ; p<.001 ; \eta^{2}=.211\right)$. In der folgenden Tabelle 3 sind die Kennwerte der besprochenen Varianzanalyse abgetragen. 
Tabelle 3: Die Symptombelastung in Jugendalter und jungem Erwachsenenalter im Vergleich über die drei Gruppen mit verschieden wahrgenommenen Vatertypen

\begin{tabular}{|c|c|c|c|c|c|c|c|c|c|c|}
\hline \multirow[t]{2}{*}{ Skala (Alter) } & \multicolumn{2}{|c|}{$\begin{array}{c}\text { Vater } \\
\text { distanziert } \\
(1) \\
(\mathrm{N}=11)\end{array}$} & \multicolumn{2}{|c|}{$\begin{array}{c}\text { Vater } \\
\text { normativ } \\
(2) \\
(\mathrm{N}=174)\end{array}$} & \multicolumn{2}{|c|}{$\begin{array}{c}\text { Vater } \\
\text { negativ } \\
(3) \\
(\mathrm{N}=28)\end{array}$} & \multirow[t]{2}{*}{$\mathbf{F}$} & \multirow[t]{2}{*}{$p$} & \multirow[t]{2}{*}{$\eta^{2}$} & \multirow[t]{2}{*}{$\begin{array}{l}\text { Post-hoc } \\
\text { (Bon- } \\
\text { ferroni) }\end{array}$} \\
\hline & $M$ & $S D$ & $M$ & $S D$ & $M$ & $S D$ & & & & \\
\hline Externalisierende Auffälligkeiten (14) & 13.73 & 4.47 & 12.06 & 5.72 & 15.71 & 5.86 & 5.22 & .006 & .047 & $3>2$ \\
\hline Internalisierende Auffälligkeiten (14) & 17.09 & 7.30 & 12.37 & 7.30 & 16.89 & 7.58 & 6.20 & .002 & .056 & $3>2$ \\
\hline Gesamtauffälligkeiten (14) & 38.55 & 10.17 & 30.21 & 13.55 & 42.07 & 15.49 & 10.31 & $<.001$ & .089 & $3>2$ \\
\hline Externalisierende Auffälligkeiten (15) & 14.34 & 5.00 & 11.48 & 5.74 & 18.10 & 6.30 & 16.41 & $<.001$ & .135 & $3>2$ \\
\hline Internalisierende Auffälligkeiten (15) & 13.37 & 8.16 & 10.44 & 6.35 & 17.25 & 10.95 & 11.20 & $<.001$ & .096 & $3>2$ \\
\hline Gesamtauffälligkeiten (15) & 31.00 & 15.39 & 27.60 & 12.84 & 44.14 & 18.03 & 17.50 & $<.001$ & .143 & $3>1=2$ \\
\hline Externalisierende Auffälligkeiten (16) & 13.86 & 6.48 & 11.17 & 5.78 & 16.64 & 6.04 & 11.14 & $<.001$ & .096 & $3>2$ \\
\hline Internalisierende Auffälligkeiten (16) & 15.13 & 8.94 & 10.40 & 6.89 & 16.08 & 10.44 & 8.24 & $<.001$ & .073 & $3>2$ \\
\hline Gesamtauffälligkeiten (16) & 30.26 & 17.12 & 27.07 & 13.12 & 42.21 & 16.31 & 14.58 & $<.001$ & .122 & $3>1=2$ \\
\hline Externalisierende Auffälligkeiten (17) & 14.38 & 5.79 & 10.57 & 6.00 & 16.15 & 6.88 & 11.38 & $<.001$ & .098 & $3>2$ \\
\hline Internalisierende Auffälligkeiten (17) & 18.54 & 7.23 & 10.17 & 6.55 & 15.59 & 10.94 & 12.33 & $<.001$ & .105 & $3=1>2$ \\
\hline Gesamtauffälligkeiten (17) & 37.45 & 16.77 & 26.03 & 13.14 & 39.84 & 19.68 & 13.48 & $<.001$ & .114 & $3=1>2$ \\
\hline Externalisierende Auffälligkeiten (23) & 13.18 & 6.15 & 7.15 & 4.84 & 13.79 & 4.62 & 28.03 & $<.001$ & .211 & $3=1>2$ \\
\hline Internalisierende Auffälligkeiten (23) & 6.91 & 11.70 & 10.70 & 8.19 & 13.89 & 9.46 & 2.95 & .055 & .027 & n.s. $(3>1)$ \\
\hline Gesamtauffälligkeiten (23) & 35.73 & 27.83 & 36.26 & 19.72 & 61.16 & 18.77 & 18.80 & $<.001$ & .152 & $3>2=1$ \\
\hline
\end{tabular}

\section{Diskussion}

Das Jugendalter ist eine Phase der Transformation der Eltern-Kind-Beziehung, bei der das väterliche Modell entscheidend zur Autonomieentwicklung ihrer Kinder beiträgt (Steinberg 2001). Zugleich ist es eine Phase, in der entscheidende Weichen gestellt werden in Richtung auf eine psychopathologische oder eher gesunde Entwicklung im jungen Erwachsenenalter. Studien, die den Einfluss von Vätern auf die Entwicklung von Symptombelastung ihrer Kinder untersucht haben, sind generell rar und beziehen sich auf den Einfluss des Vaters auf psychische und körperliche Symptome bei Kindern und Jugendlichen (Bögels/Phares 2008); Studien, die überprüfen, ob der väterliche Einfluss auch noch im jungen Erwachsenenalter anhält, fehlen gänzlich.

Ein wichtiges Ziel der vorliegenden Studie ist daher die Analyse der Veränderungen in der Symptombelastung vom Jugend- zum jungen Erwachsenenalter bei einer Stichprobe, die während der Jugendzeit in Familien mit drei sehr unterschiedlich wahrgenommenen Vatertypen gelebt haben. Ein weiterer Aspekt ist der Geschlechtsvergleich in den verschiedenen Bereichen der Symptombelastung im Verlauf im Alter von 14 bis 23 Jahren.

Bisher gibt es nur wenige Studien, die den Entwicklungsverlauf in der Psychopathologie vom Jugend- bis zum jungen Erwachsenenalter mit den gleichen Instrumenten wie in der vorliegenden Studie untersucht haben (van Oort et al. 2009/Reef et al., 2009/Hof- 
stra et al. 2001). In unserer Studie zeigte sich in der Gruppe der jungen Erwachsenen, welche ihren Vater im Jugendalter als normativ beschrieben haben, erwartungsgemäß ein Abfall der Symptombelastung während des Jugendalters. Allerdings stiegen die Gesamtauffälligkeiten mit dem Übergang ins Erwachsenenalter, also zwischen dem Alter von 17 und 23 Jahren, an. Die internalisierende und externalisierende Symptombelastung zeigte keinen geschlechtsspezifischen Verlauf über die Zeit. Allerdings hatten die weiblichen im Vergleich zu den männlichen Probanden zu allen Zeitpunkten eine erhöhte internalisierende Symptombelastung. Der Geschlechtsunterschied scheint folglich über die Adoleszenz und bis ins junge Erwachsenenalter hinein stabil zu sein. Dies steht nur teilweise in Einklang mit unseren Hypothesen, da wir im Sinne der gender intensification (Hill/Lynch 1983) einen Abfall der Geschlechtsunterschiede zum jungen Erwachsenenalter hin erwarteten. Unsere Ergebnisse widersprechen jedoch neueren Studien, welche keine Geschlechtsunterschiede in der Adoleszenz gefunden hatten (Priess et al. 2009).

Unsere Erwartungen bezüglich des negativen Einflusses einer elterlichen Trennung auf die Symptombelastung konnten nur für das junge Erwachsenenalter bestätigt werden: Hier weisen junge Erwachsene mit 23 Jahren eine höhere externalisierende Symptombelastung auf als junge Erwachsene aus einem Haushalt mit zwei verheirateten Elternteilen.

Hinsichtlich des Einflusses der im Jugendalter verschieden wahrgenommenen Vatertypen wurden unsere Erwartungen ebenfalls teilweise bestätigt: Der von uns gefundene Verlauf in der Gruppe mit einem normativen Vatertypus (Rückgang zu Ende der Adoleszenz, Zunahme im jungen Erwachsenenalter) entspricht recht gut den Befunden von Hofstra, van der Ende und Verhulst (2001) an einer unausgelesenen Stichprobe von Probanden, die über einen Zeitraum von 10 Jahren, das heißt von der frühen Adoleszenz bis zum jungen Erwachsenenalter, mit den gleichen Instrumenten (YSR, YASR) untersucht wurden. Dieser Anstieg scheint normativ zu sein, in dem Sinne, dass mit dem Übergang ins Erwachsenenalter neuartige Entwicklungsaufgaben bewältigt werden müssen (Roisman/Masten/Coatsworth/Tellegen 2004), was zu höherer Symptombelastung führen kann. Auch unter Bedingungen einer positiven, supportiven Beziehung zum Vater ist also offenkundig der Übergang zum jungen Erwachsenenalter stressbelastet und mit erhöhter Symptombelastung verbunden.

In der Gruppe der jungen Erwachsenen, welche ihren Vater im Jugendalter als distanziert beschrieben haben, zeigt sich erstaunlicherweise ein Abfall der internalisierenden Auffälligkeiten, allerdings bleiben die Werte auf einem deutlich höheren Niveau, verglichen mit Probanden aus der Gruppe mit normativen Vätern. Im Vergleich mit den jungen Erwachsenen, welche ihren Vater im Jugendalter als normativ beschrieben haben, fällt auf, dass die jungen Erwachsenen, welche ihren Vater im Jugendalter als distanziert beschrieben haben, im Alter von 17 und 23 Jahren mehr Auffälligkeiten aufweisen. Dies macht sich im Alter von 17 Jahren in Form von internalisierenden, im Alter von 23 Jahren in Form von externalisierenden Auffälligkeiten bemerkbar. Dies spricht für die These von Reef und Kollegen (2009), dass es bei bestimmten Subgruppen eine heterotype Kontinuität gibt. Der Wechsel von einer hohen internalisierenden zu einer ausgeprägten externalisierenden Symptomatik ist problematisch, da externalisierende Störungen als sehr stabil gelten und einen wichtigen Risikofaktor für spätere Psychopathologie darstellen (Trautmann-Villalba/Gerhold/Polowczyk/Dinter-Jörg/Laucht/Esser/Schmidt 2001). Des Weiteren führen externalisierende Auffälligkeiten unter Gleichaltrigen häufig zu deviantem 
Verhalten und Delinquenz (Dishion/Patterson/Stoolmiller/Skinner 1991). Andererseits fanden wir, ebenfalls in Übereinstimmung mit Reef und Kollegen, dass es sehr unwahrscheinlich ist, dass externalisierende Probleme im Jugendalter sich in internalisierende Probleme im jungen Erwachsenenalter verändern.

Die Symptombelastung der jungen Erwachsenen, welche ihren Vater im Jugendalter als negativ beschrieben haben, ist im Vergleich zu den jungen Erwachsenen, welche ihren Vater im Jugendalter als normativ beschrieben haben, von 14 bis 23 Jahren durchgehend erhöht.

Gerade ein über das Jugendalter zunehmend negativ eingeschätzter Vater hängt folglich positiv mit der Symptombelastung seines Kindes in Jugend- und jungem Erwachsenenalter zusammen. Jugendliche, welche ihren Vater als distanziert erleben, scheinen dies bis zum Übergang ins Erwachsenenalter ausgleichen zu können.

\section{Langzeitauswirkungen}

Bezüglich der Langzeitauswirkungen des Vatertyps über die Transition des Jugendlichen ins junge Erwachsenenalter hinaus zeigt die vorliegende Studie hohe Langzeiteffekte des wahrgenommenen Vatertyps auf die Symptombelastung. Damit bestätigen sich die Ergebnisse von Aquilino (2006), welcher in seiner Längsschnittstudie zum einen Kontinuität in der Vater-Jugendlichen-Beziehung über die Transition ins Erwachsenenalter hinaus, zum anderen einen starken Zusammenhang zwischen der Involviertheit des Vaters im Jugendalter seines Kindes und der Vater-Kind-Beziehung im jungen Erwachsenenalter gefunden hat. Eine enge Bindung an den Vater überdauerte mit großer Wahrscheinlichkeit mehrere zentrale Transitionen im Leben des Heranwachsenden. Unsere Ergebnisse stehen damit ebenfalls im Einklang mit den Ergebnissen von Stewart-Brown und Kollegen (2005), welche drei Geburtskohorten (1946, 1958 und 1970) bezüglich der Eltern-Kind-Beziehung und gesundheitlicher Probleme im Erwachsenenalter untersuchten und erhebliche Langzeitfolgen feststellten (Stewart-Brown/Fletcher/Wadsworth 2005). In dieser Studie zeigten sich ebenfalls signifikant negative Zusammenhänge zwischen Problemen in der Eltern-Kind-Beziehung und der Gesundheit im Erwachsenenalter.

Es gibt allerdings auch gegenteilige Befunde. So zeigt sich in der Studie von Fanti und Kollegen (2008), welche die Auswirkungen der Vater-Jugendlichen-Beziehung nach einem Jahr untersuchte, kein Effekte der Vater-Jugendlichen-Beziehung auf die Symptombelastung. Dies deutet darauf hin, dass die Effekte der Vater-Jugendlichen-Beziehung mit einer gewissen Latenz erst nach einigen Jahren auftreten. Die distinktiven Funktionen des Vaters wie die Förderung der Autonomie unterstützen diese Interpretation, da ein Mangel in diesen Funktionen erst zu einem späteren Zeitpunkt zu dysfunktionalen Verhaltensweisen und einem damit verbundenen Anstieg an Symptombelastung führen kann (Fanti/Henrich/Brookmeyer/Kuperminc, 2008). 


\section{Limitationen der durchgeführten Studie}

Hinsichtlich der Limitationen der vorliegenden Studie ist an erster Stelle die geringe Stichprobengröße vor allem in der Gruppe der Jugendlichen, welche ihren Vater als distanziert wahrnahmen, zu nennen. Eine Kontrolle der Mutter-Jugendlichen-Beziehung sowie eventueller Geschwisterbeziehungen oder Peerbeziehungen im Jugendalter bzw. jungen Erwachsenenalter wäre aufgrund einer potentiellen Mediatorrolle ebenfalls sinnvoll. Des Weiteren fehlt eine wiederholte Erhebung des wahrgenommenen Vatertypus im jungen Erwachsenenalter. Durch die Langzeiterhebung im Jugendalter haben wir zwar ausgeschlossen, dass der Vatertypus vom Jugendlichen nur aufgrund seines aktuellen Entwicklungsstandes beispielsweise als negativ wahrgenommen wurde. Eine wiederholte Befragung wäre allerdings eventuell aufschlussreich, denn negative Vater-Kind-Beziehungen könnten sich mit dem Auszug des Kindes möglicherweise verbessert haben (Galambos/Kotylak, 2012). Familienstrukturelle Veränderungen, welche sich ebenfalls auf die Symptombelastung auswirken könnten, wurden von uns nicht abhängig vom Vatertyp untersucht. Dies sollte in zukünftigen Untersuchungen an größeren Studien geprüft werden. Da in der Gruppe der Probanden, welche ihren Vater im Jugendalter als distanziert beschrieben haben, ein höherer Anteil an alleinerziehenden Haushalten vertreten war, können wir die Familienstruktur als eine weitere mögliche Erklärung der wahrgenommenen väterlichen Distanziertheit nicht ausschließen. Weitere Faktoren, welche zu Verzerrungen der Analyse geführt haben können, sind die kategoriale Analyse in Form dreier Vatertypen und die geringe Anzahl an Kontrollvariablen. Die kategoriale Analyse wurde primär zur Veranschaulichung genutzt, da eine kategoriale Analyse in der Form von Vatertypen die Plastizität der Ergebnisse und damit die Implikationen für die Praxis aus unserer Sicht deutlich erhöht.

\section{Ausblick auf zukünftige empirische Forschung und Implikationen für die Praxis}

Die Vermutung, dass Auswirkungen des wahrgenommenen Vatertyps auf die Symptombelastung nicht nur im Jugendalter sondern bis ins Erwachsenenalter bestehen, wurde in dieser Arbeit bestätigt. Wenig unterstützende, negative Vater-Kind-Beziehungen hatten sehr langanhaltende Auswirkungen auf die Symptombelastung. Allerdings bedarf es dringend weiterer Forschung, um die väterlichen Einflüsse auf die Psychopathologie von Kindern genauer zu eruieren. Auf diesem Gebiet ist vor allem Bedarf an Langzeitstudien mit größeren Stichproben, um die Repräsentativität der einzelnen Gruppen gewährleisten zu können. Eine Kontrolle des Alters durch eine Betrachtung einzelner Kohorten wäre im Zusammenhang einer größeren Untersuchung ratsam, um eine Interpretation unabhängig von eventuellen Kohorteneffekten zu ermöglichen. 


\section{Literatur}

Achenbach, T. M. (1997). Young adult self-report. Burlington, VT: University of Vermont, Department of Psychiatry.

Achenbach, T. M., Becker, A., Döpfner, M., Heiervang, E., Roessner, V., Steinhausen, H.-C. \& Rothenberger, A. (2008). Multicultural assessment of child and adolescent psychopathology with ASEBA and SDQ instruments: Research findings, applications, and future directions. Journal of Child Psychology and Psychiatry, 49, S. 251-275.

Achenbach, T. M., Howell, C. T., McConaughy, S. H. \& Stanger, C. (1995). Six-year predictors of problems in a national sample: III. Transitions to young adult syndromes. Journal of the American Academy of Child and Adolescent Psychiatry, 34, S. 658-69.

Amato, P. R. \& Rivera, F. (1999). Paternal involvement and children's behavior problems. Journal of Marriage and Family, 61, S. 375-384.

Aquilino, W. S. (2006). The noncustodial father-child relationship from adolescence into young adulthood. Journal of Marriage and Family, 68, S. 929-946.

Arnett, J. J. (2004). Emerging adulthood: The winding road from the late teens through the twenties. Oxford University Press: New York.

Bögels, S. \& Phares, V. (2008). Fathers' role in the etiology, prevention and treatment of child anxiety: A review and new model. Clinical Psychology Review, 28, S. 539-558.

Booth, A., Scott, M. E. \& King, V. (2010). Father residence and adolescent problem behavior: Are youth always better off in two-parent families? Journal of Family Issues, 31, S. 585-605.

Bornstein, M. H., Hahn, C.-S. \& Haynes, O. M. (2010). Social competence, externalizing, and internalizing behavioral adjustment from early childhood through early adolescence: Developmental cascades. Development and Psychopathology, 22, S. 717-735.

Cabrera, N. J., Tamis-LeMonda, C. S., Bradley, R. H., Hofferth, S. \& Lamb, M. E. (2000). Fatherhood in the twenty-first century. Child Development, 71, S. 127-136.

Dekovic, M., Buist, K. L. \& Reitz, E. (2004). Stability and changes in problem behavior during adolescence: Latent growth analysis. Journal of Youth and Adolescence, 33, S. 1-12.

Dishion, T. J., Patterson, G. R., Stoolmiller, M., \& Skinner, M. L. (1991). Family, school, and behavioral antecedents to early adolescent involvement with antisocial peers. Developmental Psychology, 27, S. $172-180$

Döpfner, M., Plück, J., Berner, W., Fegert, J. M., Huss, M., Lenz, K., Schmeck, K., Lehmkuhl, U., Poustka, F. \& Lehmkuhl, G. (1997). Psychische Auffälligkeiten von Kindern und Jugendlichen in Deutschland - Ergebnisse einer repräsentativen Studie: Methodik, Alters-, Geschlechts- und Beurteilereffekte. Zeitschrift für Kinder- und Jugendpsychiatrie und Psychotherapie, 25, S. 218-33.

Fanti, K. A., Henrich, C. C., Brookmeyer, K. A. \& Kuperminc, G. P. (2008). Toward a transactional model of parent-adolescent relationship quality and adolescent psychological adjustment. Journal of Early Adolescence, 28, S. 252-276.

Finkelhor, D., Ormrod, R. K. \& Turner, H. A. (2007). Poly-victimization: A neglected component in child victimization. Child Abuse \& Neglect, 31, S. 7-26.

Flouri, E. \& Buchanan, A. (2003). The role of father involvement in children's later mental health. Journal of Adolescence, 26, S. 63-78.

Furman, W. \& Buhrmester, D. (1985). Children's perceptions of the personal relationships in their social networks. Developmental Psychology, 21, S. 1016-1024.

Galambos, N. L., Barker, E. T. \& Almeida, D. M. (2003). Parents do matter: Trajectories of change in internalizing and externalizing problems in early adolescence. Child Development, 74, S. 578-594.

Galambos, N. L. \& Kotylak, L. A. (2012). Transformations in parent-child relationships from adolescence to adulthood. In: Laursen, B. \& Collins, W. A. (Hrsg.), Relationship pathways: From adolescence to young adulthood. Thousand Oaks, CA: Sage, S. 23-42.

Galambos, N. L. \& Krahn, H. J. (2008). Depression and anger trajectories during the transition to adulthood. Journal of Marriage and the Family, 70, S. 15-27. 
Grant, K. E., Compas, B. E., Thurm, A. E., McMahon, S. D., Gipson, P. Y., Campbell, A. J., Krochock, K. \& Westerholm, R. I. (2006). Stressors and child and adolescent psychopathology: Evidence of moderating and mediating effects. Clinical Psychology Review, 26, S. 257-283.

Hampel, P. \& Pössel, P. (2012). Psychische Auffälligkeiten und Stressverarbeitung im Jugendalter: Eine 2-Jahres-Kohorten-Sequenz-Studie. Zeitschrift für Gesundheitspsychologie, 20, S. 3-12.

Hill, J. P. \& Lynch, M. E. (1983). The intensification of gender-related role expectations during early adolescence. In: Brooks-Gunn, J. \& Petersen, A. C. (Hrsg.), Girls at puberty: Biological and psychosocial perspectives. New York: Plenum, S. 201-228.

Hofstra, M. B., van der Ende, J. \& Verhulst, F. C. (2001). Adolescents' self-reported problems as predictors of psychopathology in adulthood: 10-year follow-up study. The British Journal of Psychiatry, 179, S. 203-209.

Lieb, R., Isensee, B., Höfler, M., Pfister, H. \& Wittchen, H. U. (2002). Parental major depression and the risk of depression and other mental disorders in offspring: A prospective-longitudinal community study. Archives of General Psychiatry, 59, S. 365-74.

Masten, A. S., Roisman, G. I., Long, J. D., Burt, K. B., Obradovic, J., Riley, J. R., Boelcke-Stennes, K. \& Tellegen, A. (2005). Developmental cascades: Linking academic achievement and externalizing and internalizing symptoms over 20 years. Developmental Psychology, 41, S.733-46.

Matzner, M. (2007). Alleinerziehende Väter - eine schnell wachsende Familienform. In: Mühling, T. \& Rost, H. (Hrsg), Väter im Blickpunkt. Verlag Barbara Budrich: Opladen, S. 225-238.

Muthén, L. K. \& Muthén, B. O. (2006). Mplus user's guide. Los Angeles: Muthén \& Muthén (4. Auflage).

Phares, V. \& Compas, B. E. (1992). The role of fathers in child and adolescent psychopathology: Make room for daddy. Psychological Bulletin, 111, S. 387-412.

Phares, V., Renk, K., Duhig, A. M., Fields, S. \& Sly, J. (2009). Gender differences in positive and negative feelings between adolescents and their fathers and mothers. Journal of Child and Family Studies, 18 , S. 213-218.

Priess, H. A., Lindberg, S. M. \& Hyde, J. S. (2009). Adolescent gender-role identity and mental health: Gender intensification revisited. Child Development, 80, S. 1531-1544.

Reef, J., Diamantopoulou, S., van Meurs, I., Verhulst, F. \& van der Ende, J. (2009). Child to adult continuities of psychopathology: A 24-year follow-up. Acta Psychiatrica Scandinavica, 120, S. 230-238.

Roisman, G. I., Masten, A. S., Coatsworth, J. D. \& Tellegen, A. (2004). Salient and emerging developmental tasks in the transition to adulthood. Child Development, 75, S. 123-133.

Salmela-Aro, K., Aunola, K. \& Nurmi, J.-E. (2008). Trajectories of depressive symptoms during emergine adulthood: Antecedents and consequences. European Journal of Developmental Psychology, 5, S. 439-465.

Schulenberg, J.-E. \& Zarrett, N. R. (2006). Mental health during emerging adulthood: Continuity and discontinuity in courses, causes, and functions. In: Arnett, J. J. \& Tanner, J. L. (Hrsg.), Emerging adults in America: Coming of age in the $21^{\text {st }}$ century. Washington, DC: APA Books.

Seiffge-Krenke, I. (2000). Causal links between stressful events, coping style, and adolescent symptomatology. Journal of Adolescence, 23, S. 675-691.

Seiffge-Krenke, I. (2001). Neuere Ergebnisse der Vaterforschung. Sind Väter notwendig, überflüssig oder sogar schädlich für die Entwicklung ihrer Kinder? Der Psychotherapeut, 46, S. 391-397.

Seiffge-Krenke, I. (2006). Leaving home or still in the nest? Parent-child relationships and psychological health as predictors of different leaving home patterns. Developmental Psychology, 42, S. 864-76.

Seiffge-Krenke, I. (2011). Coping with relationship stressors: A decade review. Journal of Research on Adolocence, 21, 196-210.

Seiffge-Krenke, I. (2012). Mehr Liebe und weniger Gewalt? Veränderungen von Vaterschaft und ihre Konsequenzen für die Kindesentwicklung und die therapeutische Arbeit. Psychotherapeut, 57, S. $148-160$.

Seiffge-Krenke, I., Overbeek, G. \& Vermulst, A. (2010). Parent-child relationship trajectories during adolescence: Longitudinal associations with romantic outcomes in emerging adulthood. Journal of Adolescence, 33, S. 159-171. 
Sheidow, A. J., Strachan, M. K., Minden, J. A., Henry, D. B., Tolan, P. H. \& Gorman-Smith, D. (2008). The relation of antisocial behavior patterns and changes in internalizing symptoms for a sample of inner-city youth: Comorbidity within a developmental framework. Journal of Youth and Adolescence, 37, S. 821-829.

Shin, Y.-M., Sung, M.-J., Lim, K.-Y., Park, K.-S. \& Cho, S.-M. (2011). The pathway of internalizing and externalizing problems from childhood to adolescence: A prospective study from age 7 to 14 16 in Korea. Community Mental Health Journal, 48, S. 384-391.

Shulman, S., Kalnitzki, E. \& Shahar, G. (2009). Meeting developmental challenges during emerging adulthood: The role of personality and social resources. Journal of Adolescent Research, 24, S. 242267.

Shulman, S. \& Noy-Sever, L. (2006). The distinctive relationships of emerging adult sons with their mothers and fathers and the attainment of age-related tasks: a qualitative study. Paper presented at the 11th Biennial Meeting of the Society for Research on Adolescence, San Francisco.

Shulman, S. \& Seiffge-Krenke, I. (1997). Fathers and adolescents. Developmental and clinical perspectives. London: Routledge.

Slep, A. M. \& O'Leary, S. G. (2005). Parent and partner violence in families with young children: Rates, patterns, and connections. Journal of Consulting and Clinical Psychology, 73, S. 435-44.

Sontag, L. M., Graber, J. A. \& Clemans, K. H. (2011). The role of peer stress and pubertal timing on symptoms of psychopathology during early adolescence. Journal of Youth and Adolescence, 40, S. 1371-1382.

Sourander, A. \& Helstelä, L. (2005). Childhood predictors of externalizing and internalizing problems in adolescence. European Child and Adolescent Psychiatry, 14, S. 415-423.

Steinberg, L. (2001). We know some things: Parent-adolescent relationships in retrospect and prospect. Journal of Research on Adolescence, 11, S. 1-19.

Stewart-Brown, S. L., Fletcher, L. \& Wadsworth, M. E. J. (2005). Parent-child relationships and health problems in adulthood in three UK national birth cohort studies. European Journal of Public Health, 15, S. 640-646.

Stolz, H. E., Barber, B. K. \& Olsen, J. A. (2005). Toward disentangling fathering and mothering: An assessment of relative importance. Journal of Marriage and Family, 67, S. 1076-1092.

Tanner, J. L., Reinherz, H. Z., Beardsea, W. R., Fitzmaurice, G. M., Leis, J. A. \& Berger, S. R. (2007). Change in prevalence of psychiatric disorders from ages 21 to 30 in a community sample. Journal of Nervous and Mental Diseases, 195, S. 298-306.

Trautmann-Villalba, P., Gerhold, M., Polowczyk, M., Dinter-Jörg, M., Laucht, M., Esser, G. \& Schmidt, M. H. (2001). Mutter-Kind-Interaktion und externalisierende Störungen bei Kindern im Grundschulalter. Zeitschrift für Kinder und Jugendpsychiatrie und Psychotherapie, 29, S. 263-73.

van Oort, F. V. A., Greaves-Lord, K., Verhulst, F. C., Ormel, J. \& Huizink, A. C. (2009). The developmental course of anxiety symptoms during adolescence: The TRAILS study. Journal of Child Psychology and Psychiatry, 50, S. 1209-1217.

Wittchen, H.-U., Perkonigg, A., Lachner, G. \& Nelson, C. B. (1998). Early Developmental Stages of Psychopathology Study (EDSP): Objectives and design. European Addiction Research, S. 18-27.

Eingereicht am/submitted on: 17.07.2012

Angenommen am/accepted on: 21.01.2013 
Anschrift des Autors und der Autorin/Address of the authors:

Fabian Escher, Diplom-Psychologe (Korrespondenzautor/Corresponding author)

Prof. Dr. Inge Seiffge-Krenke

Johannes Gutenberg-Universität Mainz

Psychologisches Institut

Abteilung für Entwicklungs- und Pädagogische Psychologie

Wallstraße 3

55099 Mainz

Deutschland/Germany

E-Mail: escherfa@uni-mainz.de seiffge@uni-mainz.de 